\title{
Arquitetura de um sistema tutor inteligente para recomendação personalizada de objetos de aprendizagem considerando a teoria dos mapas de conhecimentos estruturados
}

\author{
Architecture of an intelligent tutor system for personalized recommendation of learning objects \\ considering the theory of structured knowledge maps \\ Arquitectura de un sistema tutor inteligente para la recomendación personalizada de objetos de \\ aprendizaje considerando la teoría de mapas del conocimiento estructurados
}

Recebido: 24/11/2021 | Revisado: 28/11/2021 | Aceito: 10/12/2021 | Publicado: 17/12/2021

\author{
Sara Luzia de Melo \\ ORCID: https://orcid.org/0000-0003-3290-9115 \\ Universidade Federal de Uberlândia, Brasil \\ E-mail: saraluziamelo@gmail.com \\ Régis Michel dos Santos Sousa \\ ORCID: https://orcid.org/0000-0001-8782-0391 \\ Universidade Federal de Uberlândia, Brasil \\ E-mail: regismaicon@gmail.com \\ Luciano Vieira Lima \\ ORCID: https://orcid.org/0000-0003-0298-5467 \\ Universidade Federal de Uberlândia, Brasil \\ E-mail: lucianovieiralimaster@gmail.com
}

\begin{abstract}
Resumo
Este trabalho propõe uma arquitetura de um Sistema Tutor Inteligente baseada na Teoria dos Mapas de Conhecimentos Estruturados para personalização dos objetos de aprendizagem oferecidos ao estudante. Para isto, pretende que a interface do professor seja inserida na arquitetura do sistema. O sequenciamento adaptativo do curso é realizado por meio da teoria dos Mapas de Conhecimentos Estruturados, no qual o professor será responsável por especificar os conceitos e conhecimentos mínimos necessários para a compreensão de cada item da matriz curricular. Na interface do estudante, com o objetivo de evitar a sobrecarga cognitiva, o sistema efetuará o mapeamento das dúvidas de prérequisitos de conceitos e conhecimentos e, em seguida, apresentará os objetos de aprendizagem, em diferentes formatos, de acordo com às necessidades de aprendizagem do estudante. Desse modo, por meio das informações contidas no Modelo do Estudante e no Modelo de Domínio, ao detectar que o estudante se encontra em um ciclo improdutivo de aprendizagem o Módulo Pedagógico executará instruções personalizadas ao conhecimento prévio e perfil afetivo do estudante.
\end{abstract}

Palavras-chave: Sistemas tutores inteligentes; Computação afetiva; Planejamento adaptativo do curso; Informática na educação.

\begin{abstract}
This work proposes an architecture of an Intelligent Tutor System based on the Theory of Structured Knowledge to customize the learning objects offered to the student. For this, it intends that the teacher's interface be inserted in the system's architecture. The adaptive sequencing of the course is carried out through the theory of Structured Knowledge Maps, in which the teacher will be responsible for specifying the concepts and minimum knowledge needed to understand each item in the curriculum. In the student interface, in order to avoid cognitive overload, the system will map the prerequisite doubts of concepts and knowledge and then present the learning objects, in different formats, according to the needs of student learning. Thus, through the information contained in the Student Model and the Domain Model, upon detecting that the student is in an unproductive learning cycle, as well as a need for learning, the Pedagogical Module will execute personalized instructions based on prior knowledge and affective profile of the student.
\end{abstract}

Keywords: Intelligent tutoring systems; Affective computing; Adaptive course planning; Informatics in education.

\section{Resumen}

Este trabajo propone una arquitectura de un Sistema Tutor Inteligente basado en la Teoría de Mapas Estructurados del Conocimiento y el Método de Memorización Exponencial y Efectiva en Base Binaria para personalizar los objetos de 
aprendizaje ofrecidos al alumno. Para ello, se pretende que la interfaz del profesor se inserte en la arquitectura del sistema. La secuencia adaptativa del curso se lleva a cabo a través de la teoría de Mapas Estructurados de Conocimiento, en la que el docente se encargará de concretar los conceptos y conocimientos mínimos necesarios para comprender cada ítem del plan de estudios. En la interfaz del alumno, para evitar la sobrecarga cognitiva, el sistema mapeará las dudas prerrequisito de conceptos y conocimientos y luego presentará los objetos de aprendizaje, en diferentes formatos, de acuerdo con las necesidades de aprendizaje del alumno. Así, a través de la información contenida en el Modelo de Alumno y el Modelo de Dominio, al detectar que el alumno se encuentra en un ciclo de aprendizaje improductivo, así como una necesidad de aprendizaje, el Módulo Pedagógico ejecutará instrucciones personalizadas basadas en conocimientos previos y perfil afectivo del alumno.

Palabras clave: Sistemas de tutoría inteligente; Computación afectiva; Planificación adaptativa del curso; Informática en la educación.

\section{Introdução}

Sistemas Tutores Inteligentes (STI) são aplicações educacionais que utilizam técnicas de Inteligência Artificial com a finalidade de fornecer instruções personalizadas ao estudante e, consequentemente, proporcionar uma solução eficiente aos problemas de ensino e aprendizagem. A arquitetura clássica de um STI, conforme exposta por (Sani \& Aris, 2014), é composta por três módulos principais, sendo eles, Modelo do Estudante (características individuais do aprendiz), Módulo Pedagógico (como ensinar) e Modelo de Domínio (o que ensinar). Além disso, possui o ambiente de interação usuário/sistema, denominado Módulo Interface, responsável pela interação do sistema com o estudante. Um estudo feito no trabalho de (Dermeval et al., 2018) observou que pesquisas sobre STI se concentram na modelagem do Modelo do Estudante e/ou do Módulo Pedagógico. A modelagem dos STI tem como principal objetivo adaptar o conteúdo de aprendizagem de acordo com as preferências dos estudantes, estilos de aprendizagem, nível de conhecimento, dentre outras características especificadas no Modelo do Estudante (Brusilovsky, 2003). Contudo, alguns ambientes computacionais de aprendizagem possuem problemas de inconsistência nos conjuntos de regras do modelo de adaptação, resultando, por exemplo, em falhas conceituais na sequência dos objetos de aprendizagem oferecidos ao estudante. Sendo assim, os STI necessitam incluir métodos com a finalidade de efetuar a determinação e o gerenciamento dos conteúdos educacionais (Karampiperis \& Sampson, 2005), (Venugopalan et al., 2016).

Um sistema adaptativo eficaz precisa evitar caminhos únicos de aprendizagem (Nafea et al., 2018). Para isto, recomenda-se apresentar uma sequência de materiais de aprendizagem, em ordem apropriada, correspondente às necessidades e ao conhecimento do estudante. O Sequenciamento Curricular Adaptativo (SCA) possibilita indicar um caminho de aprendizagem personalizado ao estudante. Entretanto, o problema do SCA apresenta algumas questões, dentre as quais destacam-se: ajustar o aprendizado conforme o conhecimento prévio do estudante, como também compreender uma sequência de conteúdo, por meio dos conceitos definidos, conforme o perfil e nível de habilidade de cada estudante (Dwivedi et al., 2018), (Aquino et al., 2020). Além da adaptação dos recursos educacionais, a construção de um ambiente propício à aprendizagem necessita integrar à arquitetura do STI informações referentes aos aspectos emocionais, motivacionais e pedagógicos (Rodrigues \& Carvalho, 2005). Isto se deve ao fato de que as emoções interferem diretamente no processo de ensino e aprendizagem. Estudantes que se encontram em uma emoção prejudicial ao aprendizado, não conseguem assimilar, da melhor maneira possível, o conteúdo que lhes é proposto. No entanto, para (Chabot et al., 2005) e (Dweck, 2013), estudantes que se encontram em uma emoção favorável ao aprendizado têm um maior interesse no conteúdo de aprendizagem, como também ocorre um aumento na memorização dos itens compreendidos.

A personalização de ambientes computacionais de aprendizagem está entre as áreas de pesquisa mais importantes na educação baseada em computador. Todavia, estes ambientes devem rastrear, com precisão, as atividades dos estudantes, monitorar suas características e elaborar intervenções e/ou estímulos conforme estratégias pedagógicas eficientes. Desse modo, além adaptação do material de aprendizagem, julga-se necessário fornecer instruções adaptadas ao estudante, considerando suas características individuais, responsáveis por otimizar o ganho de aprendizagem (Woolf, 2010), (Narciss et al., 2014), (Truong, 
2016).

\section{Fundamentação Teórica}

Esta seção apresenta os conceitos teóricos para compreender a abordagem proposta neste trabalho. As Seções 2.1 e 2.2 descrevem os métodos utilizados para a recomendação personalizada dos objetos de aprendizagem de acordo com o conhecimento prévio do estudante.

\subsection{Mapas de Conhecimentos Estruturados}

A Teoria das Categorias (Eilenberg; Maclane, 1945) possibilita levantar questões sobre epistemologia e ontologias matemáticas. Sendo assim, é uma generalização da teoria dos conjuntos. A metodologia dos Mapas de Conhecimentos Estruturados (MCE) baseia-se matematicamente na Teoria das Categorias e Funtores. Assim, a estrutura dos MCE, conforme demonstrada por (Filho, 2013), é composta pelas seguintes definições:

1. Categorias: indivíduos envolvidos nos processos;

2. Objetos: conceitos e definições existentes no domínio que deseja ensinar;

3. Morfismos: métodos, ações, aplicações de como manipular os conceitos e definições que estão envolvidos no processo de transmissão do conhecimento em questão;

4. Funtores: efetiva a adaptação dos objetos e morfismos preexistentes na categoria estudante para adquirir ou transformar um novo conhecimento.

Neste contexto, para uma construção efetiva do conhecimento durante o processo de ensino e aprendizagem, a metodologia MCE propõe efetuar um mapeamento da categoria do professor na categoria do estudante, através do Funtor. Contudo, durante a transmissão do conhecimento, preservam-se as estruturas da categoria estudante. Além disso, vale ressaltar que, nesta metodologia, conceitos são palavras que não carecem de ações para serem assimiladas, como por exemplo, definições, verbetes em um dicionário, enciclopédia ou afins. No entanto, os conhecimentos são os morfismos que são transmitidos como ações para alcançar os objetivos do curso. Segundo (Filho, 2013), para implementar um Funtor entre duas categorias, deve-se cumprir a exigência mínima de que as duas categorias possuam objetos e morfismos equivalentes. O objetivo do Funtor é, então, garantir a transmissão do conhecimento (aprendizagem) entre as categorias (professor e estudante). Para isto, o ideal é que o conhecimento inicial seja o mais básico possível. A transferência do conhecimento será realizada quando o estudante possuir uma subcategoria com objetos e morfismos mínimos, estabelecidos pelo professor, e uma base sólida para que o Funtor se estabeleça entre o estudante e o professor e, consequentemente, promova a efetivação do aprendizado (Costa et al., 2016). Além disso, os MCE possuem uma ferramenta de avaliação, denominada Tabela de Avaliação Qualitativa (TAQ), responsável pela identificação dos pré-requisitos de objetos (conceitos e definições) e morfismos (conhecimentos) que possam existir na categoria estudante. Durante a transmissão do conhecimento pode haver dúvidas em relação aos conceitos e conhecimentos, esta etapa é denominada área da ignorância, ilustrada na Figura 1, onde o raio representa o conhecimento e a área representa as dúvidas que este raio desperta no estudante. 
Figura 1 - Novo conhecimento implica em nova área de ignorância na estrutura dos MCE.

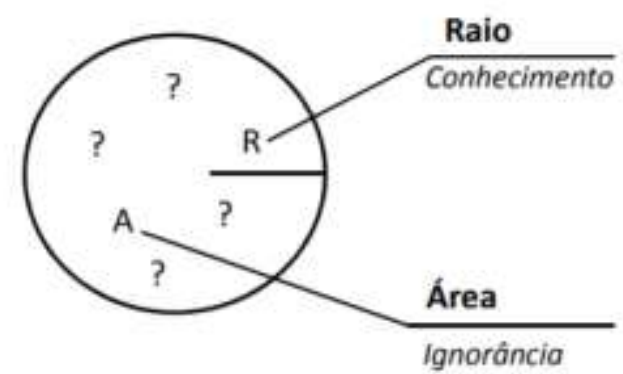

Fonte: Adaptado de Ferreira et al. (2014).

Na Figura 1, é possível observar que o raio de conhecimento determina o conteúdo a ser ensinado ao estudante. Este conteúdo exige conceitos que precisam ser adquiridos antes de aumentar o raio de conhecimento e avançar o conteúdo. As dúvidas do estudante são consideradas como pontos de ignorância que representa a ausência de determinado conhecimento ou conceito. Assim, faz-se necessário um levantamento sobre o nível de informação existente na categoria receptora do conteúdo (Costa et al., 2016). Basicamente, na estrutura dos MCE, para cada novo conhecimento (estabelecido pelo Funtor) define os conceitos e ferramentas do aprendiz (objetos), bem como o domínio do uso de tais ferramentas básicas (morfismos). Se na categoria do estudante faltar um objeto (desconhecimento de conceitos) ou um morfismo (saber manipular os objetos), a aplicação do Funtor (transmissão do novo conhecimento) não terá sucesso (Filho, 2013).

Dessa maneira, ao utilizar os MCE o estudante nunca avança no aprendizado de um novo conhecimento sem que antes não tenha aprendido o conhecimento precedente. A passagem do conhecimento, segundo (Costa et al., 2016), só será efetivada quando o estudante tiver, em uma subcategoria de si mesmo, os mesmos objetos e morfismos existentes na categoria professor, relacionada apenas ao que se deseja ensinar no momento. Satisfazendo tal exigência, um Funtor pode ser estabelecido entre as categorias promovendo o mapeamento correto dos conhecimentos entre professor e estudante e, em seguida, a transferência de um novo conhecimento.

\subsection{Método de Memorização Exponencial Efetivo na Base Binária}

Aprender novos conhecimentos e memorizá-los pode ser um grande desafio para a capacidade humana. A teoria de (Miller, 1956) indica que a memória do ser humano tem a capacidade de armazenar no máximo entre 5 a 9 informações a curto prazo. Segundo (Miller, 1956), quando há excesso de informação tem-se maior probabilidade de cometer erros e má retenção do conhecimento. No entanto, para (Cowan, 2001), o limite de armazenamento de informação, que não comprometam o raciocínio e a aprendizagem, não deve ultrapassar de 3 a 5 informações ao mesmo tempo.

Seguindo os MCE, o processo cognitivo identifica as variáveis escondidas (dúvidas) de cada estudante, avança um conhecimento por vez e, como resultado, garante que não haja a sobrecarga cognitiva (excesso de informação). Assim, obedecendo o limite de informação exposto por (Miller, 1956), o estudante poderá receber mais de uma informação ao mesmo tempo, no entanto, é necessário que apenas uma informação seja relacionada a cognição, e o restante da informação seja apenas recordação de algo já consolidado pelo estudante. A curva do esquecimento de (Ebbinghaus, 1964) constata e demostra uma forte correlação entre aprendizado, memória e tempo. A fórmula matemática de Ebbinghaus, descreve a taxa do esquecimento, e como isto ocorre após ter conhecido um determinado conteúdo e/ou informação (Chun; Heo, 2018). A redução do conhecimento em relação ao tempo, conforme ilustrada na Figura 2, representa a curva do esquecimento quando não há mais contato com o conteúdo compreendido. 
Figura 2 - Curva do esquecimento e a retenção do conhecimento em relação ao tempo.

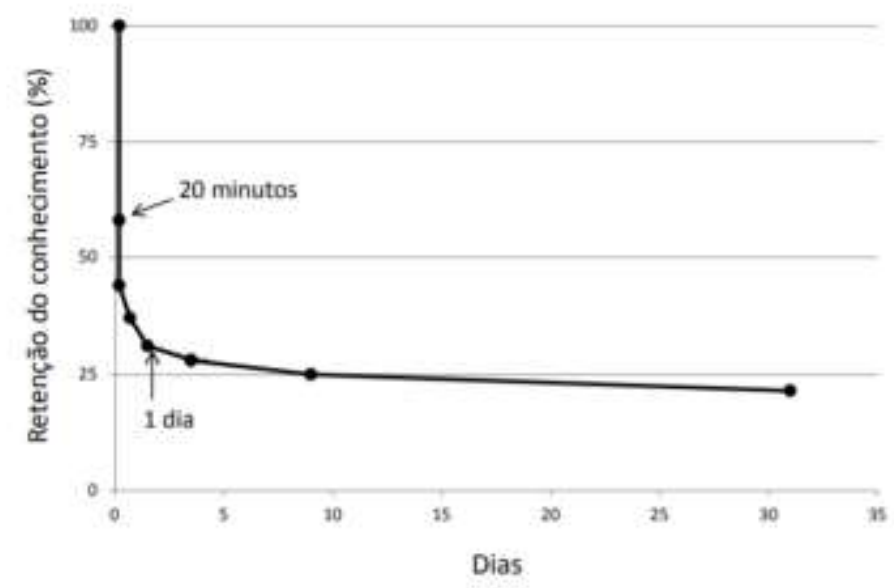

Fonte: Adaptado de Ferreira et al. (2014).

Na Figura 2, observa-se que a retenção do conhecimento diminui praticamente em $50 \%$ após vinte minutos da emissão do conhecimento. Ainda na Figura 2, note-se que após 31 dias, no melhor dos casos, o indivíduo permanece apenas com 21\% do conhecimento compreendido. Dessa forma, para manter uma taxa significativa da retenção do conhecimento, deve-se aplicar técnicas de memorização no processo de ensino e aprendizagem.

Assim sendo, o Método de Memorização Exponencial Efetivo da Base Binária (MMEEBB) fundamenta-se o tempo necessário para recordação de um conhecimento adquirido. No MMEEBB, propõe-se um reforço conceitual em intervalos de tempo, em dias, para garantir que o conhecimento seja memorizado pelo estudante (categoria receptora). Segundo (Costa et al., 2016), a cada processo de recordação, há um decréscimo de $20 \%$ no esforço em reaprender. Se em um primeiro momento há um esforço cognitivo de $100 \%$, em uma primeira revisão haverá um esforço de apenas $80 \%$, em uma terceira revisão haverá um decréscimo de $20 \%$ do esforço sobre os $80 \%$. Assim, chegou-se na taxa Intervalo de Reforço do Aprendizado (IRA) que é igual a $2^{\mathrm{n}} \operatorname{com} n=[0,1,2,3 \ldots 13]$, representando os dias.

\section{STI-LINA: Um Sistema Tutor Inteligente Adaptativo ao Curso e Personalizado ao Estudante}

Esta seção apresenta-se os aspectos metodológicos para a modelagem do Sistema Tutor Inteligente, denominado Lina, para a recomendação dos recursos educacionais com base nas necessidades individuais de aprendizagem de cada estudante sobre determinado conteúdo. Além da personalização dos recursos de aprendizagem, o STI-Lina considera que as emoções interferem diretamente no processo cognitivo, assim sendo, um estudante que encontra-se em uma emoção prejudicial ao aprendizado não consegue assimilar adequadamente o conteúdo proposto.

Neste contexto, para uma aprendizagem efetiva, não basta apenas recomendar o material de aprendizagem adequado, julga-se necessário observar as emoções do estudante durante o aprendizado e, posteriormente, fornecer instruções adaptadas ao seu perfil. Isto posto, nesta abordagem inclui-se o perfil afetivo do estudante e, por meio dessas informações, a definição das estratégias pedagógicas que possibilita a regulação das emoções negativas ao aprendizado. Além disso, técnicas de memorização do conteúdo também são consideradas. Comumente, conforme exposto por (Raabe, 2005), a modelagem de sistemas adaptativos para educação não considera a participação do professor, porém, ao inserir o professor como agente ativo é possível acrescentar abordagens pedagógicas personalizadas.

Com a finalidade de definir as regras do modelo de adaptação que aborda o problema de sequenciamento dos recursos educacionais, o STI-Lina propõe a integração do módulo/interface do professor em sua arquitetura. Amparado pela metodologia 
dos Mapas de Conhecimentos Estruturados (MCE), descrita na Seção 2.1, o professor será responsável por verificar os itens da matriz curricular e determinar os conceitos (definições) e os conhecimentos (ações sobre o conceito) relacionados a compreensão de cada item. Este processo é definido como ramificação do curso/ementa. Tendo em vista as teorias de Miller (1956) e Cowan (2001), para não comprometer o raciocínio e aprendizagem, esta ramificação do item não poderá ultrapassar o total de cinco informações, em sua complexidade.

$\mathrm{Na}$ interface do estudante aqui proposta, seguindo os MCE e a sequência curricular preestabelecida na interface do professor, efetua-se o mapeamento das dúvidas de pré-requisitos de conceitos e pré-requisitos de conhecimentos. Este mapeamento é realizado pela Tabela de Avaliação Qualitativa (TAQ). Por meio da TAQ, inicializa-se o primeiro conhecimento (conteúdo do curso) a partir da ignorância zero (ausência de dúvidas), identificada na categoria do estudante, aplicando o primeiro Funtor para mapear o primeiro conhecimento que será acrescentado à categoria estudante. Dessa forma, o STI manterá um equilíbrio estável avançando para um novo conhecimento, subsequente ao conhecimento anterior, apenas após eliminar novamente as ignorâncias (dúvidas) do estudante. Assim sendo, o estudante receberá exclusivamente os recursos educacionais, em diferentes formatos, responsáveis por cobrir as dúvidas existentes, garantindo também que o estudante não consuma tempo na procura de materiais, bem como na assimilação desnecessária de conteúdos naquele momento do curso. A Figura 3 apresenta a modelagem dos processos do STI-Lina, mais especificamente a interface do professor, interface do estudante e, por fim, o perfil afetivo responsáveis pela adaptação dos recursos educacionais oferecidos a cada estudante.

Figura 3 - Modelagem de processos do STI-Lina.

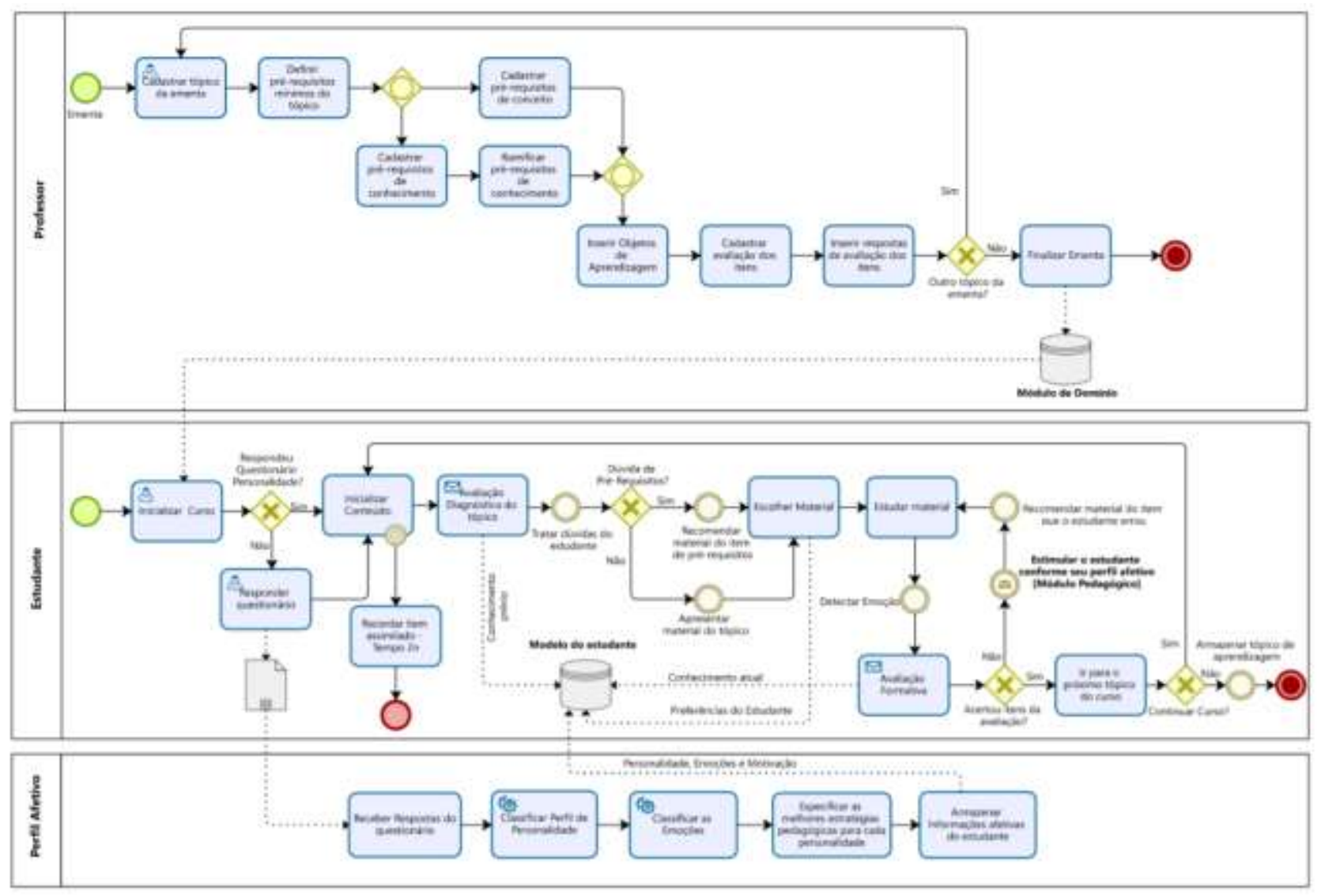

Fonte: Autores (2021).

$\mathrm{Na}$ Figura 3, ao analisar a primeira raia temos os processos do professor. Este processo inicializa-se com a ementa do curso no qual o professor seguirá para as tarefas "cadastrar tópico da ementa" e "definir os pré-requisitos mínimos". Após essas 
duas tarefas, o gateway inclusivo produz múltiplos caminhos que poderão ser seguidos no STI-Lina. Assim sendo, as tarefas "cadastrar pré-requisitos de conceitos" e "cadastrar pré-requisitos de conhecimentos" poderão ser realizadas. No entanto, no segundo gateway a próxima tarefa será realizada apenas quando as tarefas "ramificar pré-requisitos de conhecimento" e "cadastrar pré-requisitos de conceitos" forem executadas. Em seguida, o fluxo seguirá para as seguintes tarefas: "inserir objetos de aprendizagem", "cadastrar avaliação do item" e "inserir respostas de avaliação do item".

Após essas tarefas, o professor poderá retornar para a tarefa "cadastrar tópico da ementa" e repetir todos os processos para o cadastro de um novo item ou finalizar o cadastro do item precedente. Todas essas informações representam o Módulo de Domínio. O Módulo de Domínio contém toda a sequência do curso, objetos de aprendizagem de cada conteúdo, organizados conforme a teoria dos MCE, como também os itens de avaliação de cada item. Na raia do estudante, essas informações são acessadas ao inicializar a tarefa "inicializar curso". Após inicializar o curso tem-se um gateway exclusivo que avaliará se o estudante respondeu o questionário perfis de personalidade. O estudante seguirá para a próxima tarefa somente após responder o questionário solicitado. Feito isto, a tarefa "inicializar conteúdo" tem um evento de tempo responsável por verificar os intervalos da memorização do conteúdo, e, caso haja, dispara a tarefa "recordar item assimilado". Caso contrário, seguirá para a tarefa "avaliação diagnóstica" e, em seguida, dispara o evento responsável por tratar as dúvidas do estudante. A tarefa "escolher material" só será realizada após um dos eventos "recomendar material do item de pré-requisitos" ou "apresentar material do tópico" forem selecionadas. Observa-se que o fluxo seguirá para a tarefa "estudar material" que tem o evento "detectar emoções" durante o aprendizado. Em seguida, o estudante avançará para a tarefa "avaliação formativa". Se o estudante acertou os itens da avaliação, ele avança para a tarefa "ir para o próximo conteúdo do curso", caso ele não acerte o item de avaliação, são disparados dois eventos, um responsável por estimular o estudante e outro por recomendar o material adequado aos erros de aprendizagem. Este processo garante que a tarefa "ir pra o próximo tópico do curso" aconteça apenas quando tenha uma resposta positiva à tarefa "avalição formativa". As respostas do questionário perfis de personalidade serão acessadas pela tarefa "receber respostas do questionário", na raia perfil afetivo. Posteriormente, o fluxo das tarefas são "classificar perfil de personalidade", "classificar emoções", "especificar as melhores estratégias pedagógicas para cada personalidade" e "armazenar informações afetivas do estudante", respectivamente. Todas essas informações serão devolvidas para o modelo do estudante, onde são utilizadas pelo módulo pedagógico para a personalização dos aspectos motivacionais oferecidos ao estudante de determinado perfil de personalidade. O perfil afetivo do estudante integrado no STI-Lina pode ser visto em (Melo et al., 2017).

Observa-se na Figura 3 que o STI-Lina necessita desenvolver os seguintes módulos: (1) Interface do Professor; (2) Interface do Estudante; (3) Módulo gerenciador e organizador dos objetos de aprendizagem e conteúdos; (4) Módulo organizador dos itens de avaliação; (5) Módulo Classificador das Emoções (6) Módulo classificador dos Perfis de Personalidade (7) Módulo recomendação personalizada de objetos de aprendizagem (8) Módulo adaptabilidade das instruções e estratégias pedagógicas para seu funcionamento efetivo.

Assim, inicialmente, efetuou-se a validação dos aspectos teóricos dos MCE por meio da disciplina de Análise Financeira. Essa metodologia foi aplicada em nove turmas de pós-graduação, com 18 estudantes em cada. Para isto, o professor foi informado sobre os aspectos teóricos dos MCE (Seção 2.1) e, em seguida, efetuou uma ramificação de pré-requisitos de conceitos e pré-requisitos de conhecimento sobre os três tópicos da ementa, sendo eles: "Decisão do Investimento", "Matemática Financeira" e "Técnicas de Análise de Investimentos". Em seguida, realizou-se o processo de ramificação de cada conteúdo de aprendizagem, ou seja, a definição dos pré-requisitos de conceitos e pré-requisitos de conhecimentos relacionados a cada tópico da ementa. Após a aplicação dos MCE, bem como as técnicas de memorização do MMEEBB, o rendimento dos estudantes melhorou significativamente, chegando em $100 \%$ de aproveitamento no curso. Vale ressaltar que o professor não utilizou outras estratégias pedagógicas, além dos MCE e MMEEBB, que contribuíram para o ganho da aprendizagem dos estudantes. Assim 
sendo, inicializa-se o desenvolvimento dos módulos que compõem o STI-Lina, mais precisamente o Módulo do Professor e o Módulo e Interface do Estudante, apresentados a seguir.

\section{Desenvolvimento do Módulo do Professor}

Posteriormente à validação dos aspectos teóricos da metodologia dos MCE e a maximização da aprendizagem após sua utilização, o módulo Interface Professor foi desenvolvido utilizando a linguagem de programação Javascript com o framework Angular. A Interface do Professor é responsável pela organização e estrutura dos recursos de aprendizagem com base nos MCE. A Figura 4 exibe a tela inicial de cadastro da disciplina e/ou curso.

Figura 4 - Tela de cadastro da disciplina - Interface Professor.

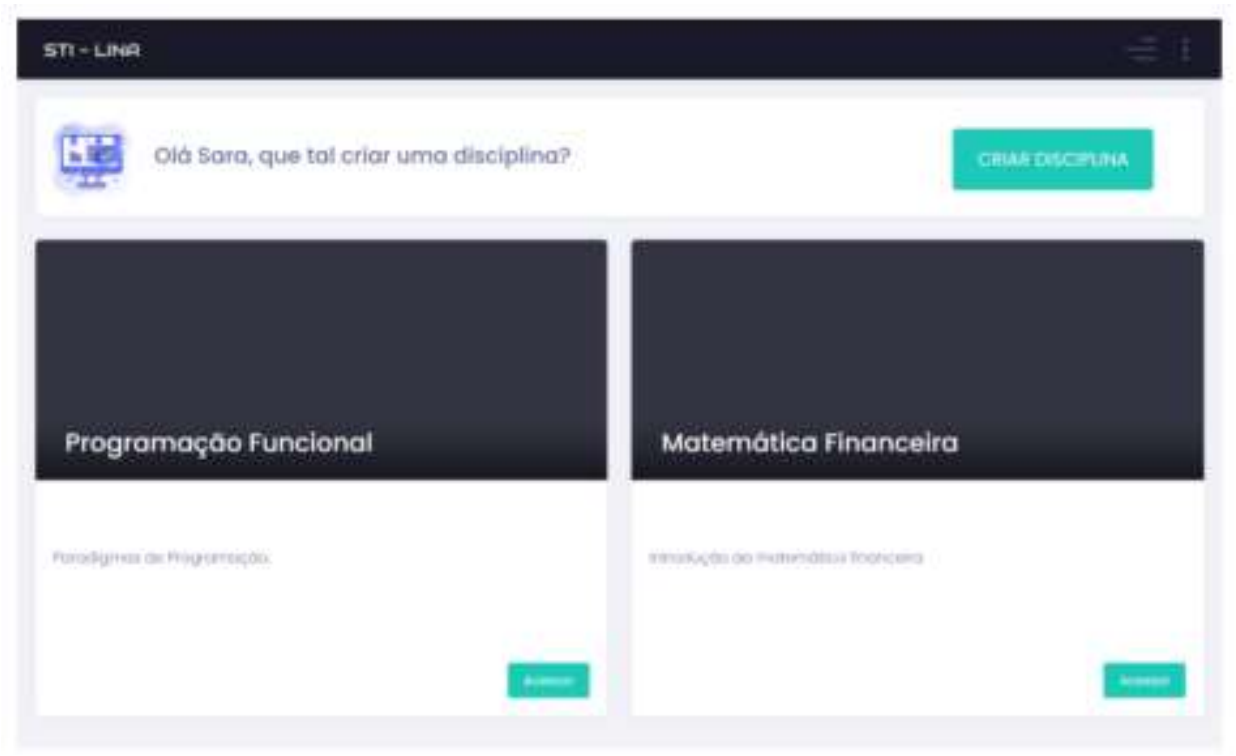

Fonte: Autores (2021)

O STI Lina foi desenvolvido conforme os processos especificados na Figura 3. Ao informar as credenciais de acesso, o professor é direcionado para a tela inicial (Figura 4), onde é possível visualizar todas as disciplinas que ele cadastrou, além da possibilidade de criar novos cursos e/ou disciplinas no ambiente computacional de aprendizagem. Assim, em todo curso, o professor deve cadastrar cada tópico da matriz curricular e, em cada ramificação, caracterizar o que é considerado como conceitos (definições) e como conhecimentos (ações sobre um conceito). Todos esses itens devem estar relacionados ao conteúdo principal do curso. Paralelamente, o professor também é responsável por inserir os recursos educacionais de cada item. A Figura 5 apresenta o processo de ramificação e inserção de conteúdos educacionais no sistema de tutoria. 
Figura 5 - Tela estruturação do conteúdo educacional - Interface Professor.

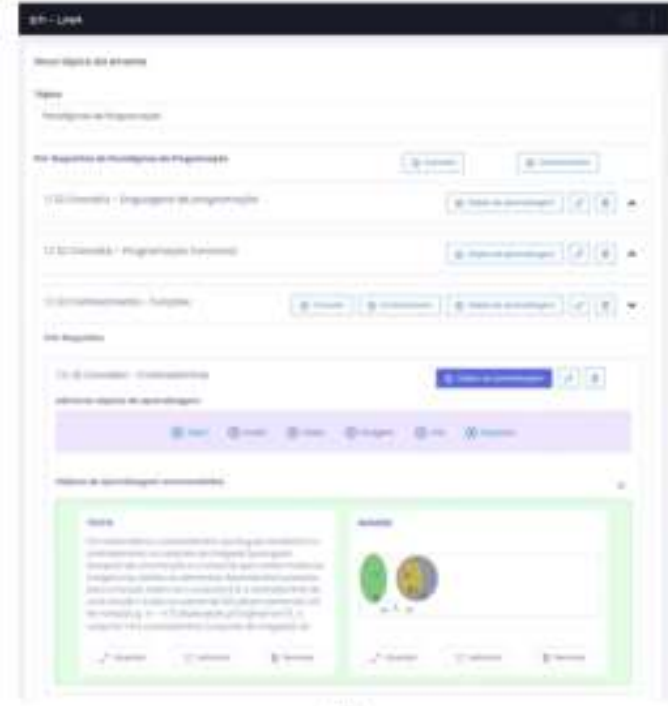

(A)

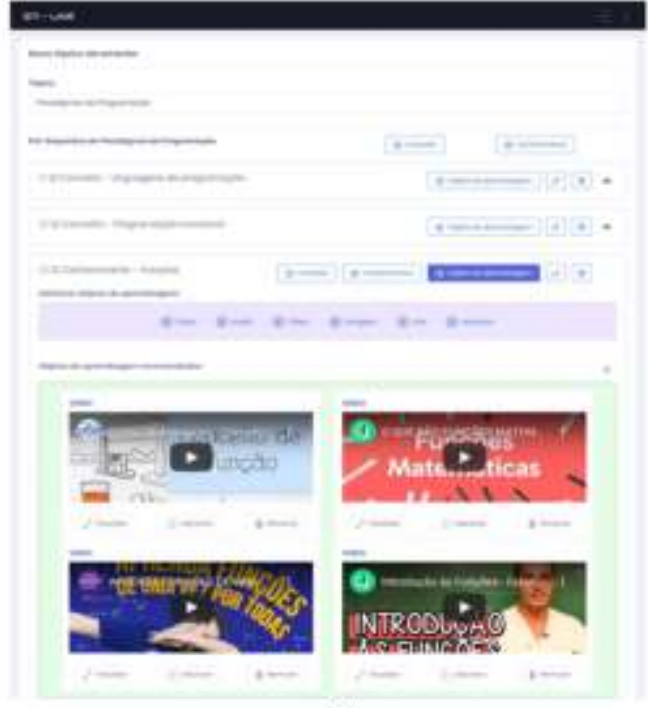

(B)

Fonte: Autores (2021).

Nesta etapa, a fim de auxiliar o professor no STI-Lina, foram integradas duas Application Programming Interface (API), Youtube API e MediaWiki API, com a finalidade de efetuar uma recomendação de possíveis recursos educacionais relacionados ao tópico da ementa. A Figura 5 apresenta a tela de ramificação da ementa, neste processo, ao selecionar um tópico da ementa como conceitos não é possível efetuar a ramificação daquele item, uma vez que, de acordo com os MCE, conceitos são apenas definições. No entanto, observa-se na Figura 5 (a) quando uma ramificação do conteúdo é definida como conhecimento, na tela de cadastro será disponível realizar outra ramificação daquele item definida como conceitos ou conhecimentos, considerando até 5 ramificações para cada tópico.

Ainda na Figura 5(a), nota-se que ao cadastrar um item como conceito o sistema realiza uma busca sobre esta definição, utilizando a MediaWiki API, e recomenda ao professor um conteúdo de textual e/ou imagem. Essa recomendação de texto devese ao fato que conceitos são apenas definições. Neste trabalho, os parâmetros da MediaWiki API foram configurados para recomendar ao professor apenas o primeiro parágrafo do texto sobre determinado conteúdo, como também a associação da recomendação a categoria do item cadastrado. Assim sendo, o sistema permite que essas recomendações sejam inseridas pelo professor, como também possibilita o professor inserir seus recursos de aprendizagem, em diferentes formatos, conforme elaboração do curso.

Contudo, ao classificar um item como conhecimento, Figura 5 (b), o sistema efetua uma recomendação de conteúdos, em formato de vídeo, utilizando a API Youtube. Neste processo, como conhecimentos são ações sobre um conceito, os parâmetros de busca da API foram definidos pela categoria educacional priorizando vídeos entre quatro até vinte minutos de duração. Assim sendo, caso retorne algum vídeo sobre determinado conteúdo de aprendizagem cadastrado, o STI-Lina recomenda os cinco primeiros vídeos, conforme sua relevância. Para cada item recomendado, o professor poderá inserir estes recursos de aprendizagem no sistema ou removê-los da plataforma. Além disso, o professor poderá inserir diferentes formatos de mídias de sua preferência que contém informações responsáveis por cobrir os pré-requisitos de conceitos e conhecimentos preestabelecidos. 


\section{Desenvolvimento da Interface do Estudante}

A modelagem do estudante proposta nesta abordagem contém informações sobre conhecimento prévio, conhecimento atual, emoções e personalidade. Todas essas informações são recuperadas na Interface do Estudante para fornecer uma recomendação adequada dos recursos educacionais e, consequentemente, promover um caminho de aprendizagem individualizado. Além disso, por meio das informações contidas no Modelo do Estudante, as estratégias e instruções pedagógicas também são adaptadas ao perfil de personalidade. Assim sendo, a Figura 5 exibe a Interface do Estudante para a recomendação dos objetos de aprendizagem personalizada às necessidades de aprendizagem do estudante.

Figura 5 - Mapeamento dos pré-requisitos de conceitos e conhecimentos.

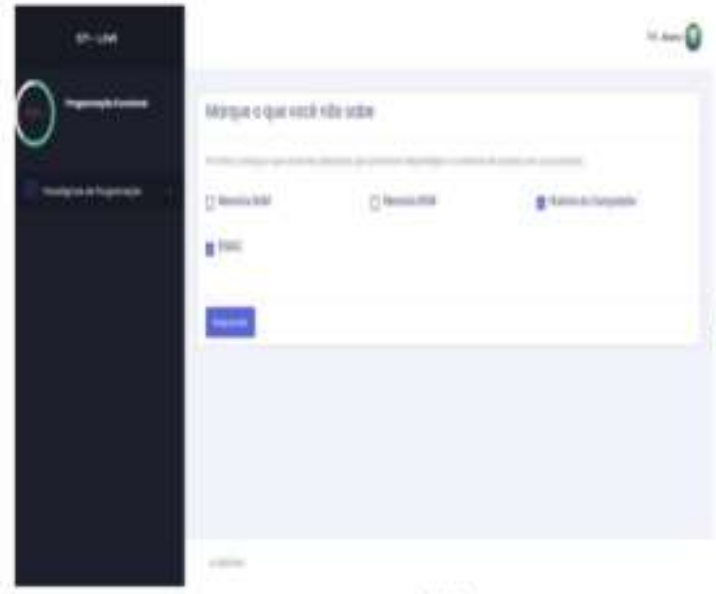

(A)
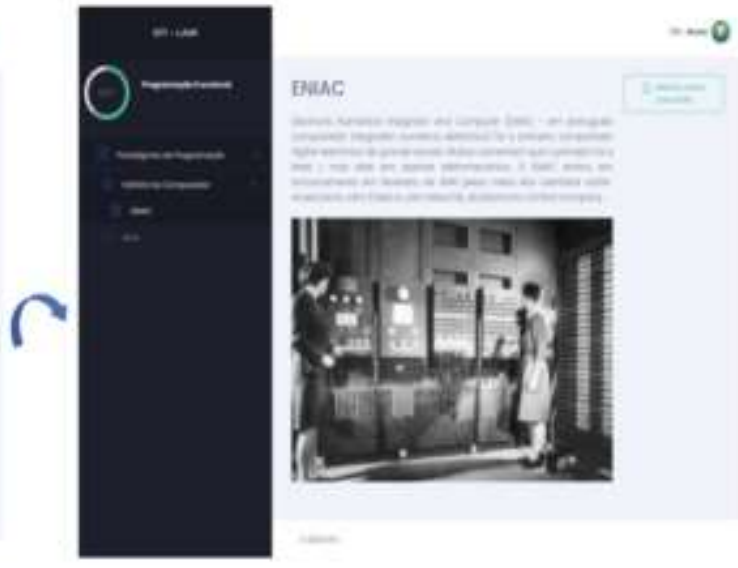

(B)

Fonte: Autores (2021).

Ao acessar o sequenciamento curricular definido na Interface do Professor, o sistema mapeará as dúvidas de prérequisitos de conceitos e conhecimentos em cada conteúdo de aprendizagem. Para isto, uma maneira de identificação dessas dúvidas sem intimidar o estudante é perguntar a ele sobre o que não sabe. Observa-se na Figura 5 (a) que o estudante tem apenas um tópico de aprendizagem, denominado paradigmas de programação, disponível para ele neste momento do curso. Por meio das definições de pré-requisitos, o estudante deverá selecionar os itens que ele desconhece. Após esta seleção, o STI Lina recomendará automaticamente os conteúdos de aprendizagem personalizados as dúvidas de cada estudante, em diferentes formatos, conforme exposto na Figura 5 (b). Assim sendo, diferentes caminhos de aprendizagem serão constituídos para cada estudante em determinado curso. Além disso, no STI-Lina, a transmissão do conhecimento inicializará a partir das folhas (conceitos), de forma botton up. Em cada item do curso, após marcar como concluída uma atividade, é disponibilizado o conteúdo. Portanto, o próximo conhecimento somente será apresentado ao estudante após verificar que não há dúvidas no conhecimento anterior.

\section{Considerações Finais e Trabalhos Futuros}

Este trabalho propôs uma arquitetura de um Sistema Tutor Inteligente (STI), amparado pedagogicamente pela teoria dos Mapas de Conhecimentos Estruturados (MCE), considerando os estados afetivos e conhecimento do estudante para efetuar o sequenciamento curricular adaptativo do curso e, consequentemente, a organização e estruturação dos conteúdos de aprendizagem relacionados a determinado curso. Todos os módulos que compõem a arquitetura clássica de um STI foram modelados, além da inserção do módulo e interface do professor na arquitetura do sistema. A teoria dos MCE foi utilizada para 
a modelagem do Módulo de Domínio, Modelo do Estudante e a modelagem da inserção da Interface do Professor a ser inserida no sistema de tutoria. Antes da implementação da Interface do Professor, um experimento foi realizado com intuito de verificar a influência dos aspectos teóricos da metodologia dos MCE no aprendizado, assim, observou-se que, após o sequenciamento curricular, proposto no MCE, houve um maior ganho da aprendizagem dos estudantes na disciplina Análise Financeira.

Nesta abordagem proposta, o STI contém oito módulos a serem desenvolvidos para seu total funcionamento. O Módulo Interface do Professor e Módulo de recomendação de conteúdos na Interface do Estudante são contribuições que foram desenvolvidas durante esta pesquisa. Considerando a ramificação de pré-requisitos de conceitos e conhecimentos, foi utilizada uma abordagem de estrutura de dados do tipo árvore para o armazenamento dos recursos educacionais. Além disso, possibilita inserir diferentes formatos de mídias sobre o mesmo conteúdo de ensino. Vale ressaltar que o STI não substitui o papel do professor, no entanto, o professor será agente ativo do processo no qual é responsável por inserir os recursos de aprendizagem, mínimos necessários, relacionados ao tópico de aprendizagem, para que o estudante não tenha dúvidas de pré-requisitos de conceitos e conhecimentos. Desse modo, temos como trabalhos futuros a implementação do módulo avaliação da aprendizagem, classificação de emoções por texto e interações de um agente pedagógico conforme perfil de personalidade.

A Interface do Professor e Estudante foram desenvolvidas conforme a modelagem proposta no STI-LINA no qual o sistema efetuará um mapeamento das dúvidas de pré-requisitos de conceitos e pré-requisitos de conhecimento antes de inicializar o conteúdo principal do curso. Conforme as dúvidas de pré-requisitos, o módulo recomendação oferece ao estudante recursos educacionais, em diferentes formatos, responsáveis por cobrir as necessidades de aprendizagem do estudante naquele instante. Dessa maneira, tem-se um ambiente educacional adaptado, considerando os recursos de aprendizagem adequados e necessários ao curso. Assim, evita-se assimilação de conteúdos prescindíveis que possam causar sobrecarga cognitiva (excesso de informação). Assim sendo, verifica-se que os aspectos metodológicos desta abordagem poderão ser utilizados tanto no ensino presencial quanto no ensino a distância.

\section{Referências}

Aquino, B. et al. (2020). Geração de sequências curriculares adaptativas baseada no perfil dos alunos e materiais didáticos utilizando o algoritmo nsga-iii. In: SBC. Anais do XXXI Simpósio Brasileiro de Informática na Educação. 902-911.

Brusilovsky, P. (1998). Methods and techniques of adaptive hypermedia. In: Adaptive hypertext and hypermedia. Springer,p. 1-43.

Chabot, D. et al. (2005). Pedagogia Emocional: sentir para aprender. Sá Editora.

Costa, N. S. et al. (2016). Teaching and learning with structured knowledge maps: An analysis about the new approach. Creative Education, Scientific Research Publishing, 7(15), 2212.

Cowan, N. (2001). The magical number 4 in short-term memory: A reconsideration of mental storage capacity. Behavioral and brain sciences, Cambridge University Press, 24(1), 87-114.

Dermeval, D. et al. (2018). Authoring tools for designing intelligent tutoring systems: a systematic review of the literature. International Journal of Artificial Intelligence in Education, Springer, 28(3), 336-384.

Dwivedi, P., Kant, V., Bharadwaj, K. K. (2018). Learning path recommendation based on modified variable length genetic algorithm. Education and information technologies, Springer, 23(2), 819-836.

Ebbinghaus, H. (1964). Memory: Acontribution to experimental psychology. Dover.

Eilenberg, S., Maclane, S. (1945). General theory of natural equivalences. Transactions of the American Mathematical Society, American Mathematical Society, 58(2), 231-294.

Ferreira, D. C. M. et al. (2014). Mapas de conhecimento estruturado: proposta de uma nova abordagem metodológica de ensino e aprendizagem. Educare, 9.

Filho, R. B. (2013). Uma abordagem para ensino baseada na teoria da aprendizagem significativa utilizando a teoria das categorias. Tese (Doutorado) Universidade Federal de Uberlândia - Departamento de Engenharia Elétrica.

Karampiperis, P., Sampson, D. (2005). Adaptive learning resources sequencing in educational hypermedia systems. Journal of Educational Technology \& Society, JSTOR, 8(4), 128-147. 
Research, Society and Development, v. 10, n. 16, e518101623831, 2021

(CC BY 4.0) | ISSN 2525-3409 | DOI: http://dx.doi.org/10.33448/rsd-v10i16.23831

Melo, S. L. et al. (2017). Modelo do estudante baseado em emoções e perfis de personalidade para recomendação de estratégias pedagógicas personalizadas. In Brazilian Symposium on Computers in Education (Simpósio Brasileiro de Informática na Educação-SBIE) 28(1), 967.

Miller, G. A. (1956). The magical number seven, plus or minus two: Some limits on our capacity for processing information. Psychological review, American Psychological Association, 63(2), 81, 1956.

Narciss, S. et al. (2014). Exploring feedback and student characteristics relevant for personalizing feedback strategies. Computers \& Education, Elsevier, 71, $56-76$.

Rodrigues, L. M. L., \& Carvalho, M. (2005). Sti-i: Sistemas tutoriais inteligentes que integram cognição, emoção e motivação. Revista Brasileira de Informática na Educação, 13(1), 20-34.

Sani, S., aris, T. N. (2014) Computational intelligence approaches for student/tutor modelling: A review. In: IEEE. 2014 5th International Conference on Intelligent Systems, Modelling and Simulation. 72-76.

Truong, H. M. (2016). Integrating learning styles and adaptive e-learning system: Current developments, problems and opportunities. Computers in human behavior, Elsevier, 55, 1185-1193.

Venugopalan, S., Srinath, M., Rodrigues, P. (2016). Recommender system for e-learning through content and profile based approach. In: Proceedings of the Second International Conference on Information and Communication Technology for Competitive Strategies. 1-5.

Woolf, B. (2010). Building intelligent interactive tutors: Student-centered strategies for revolutionizing e-learning. Morgan Kaufmann. 